\title{
MORN motifs in plant PIPKs are involved in the regulation of subcellular localization and phospholipid binding
}

\author{
Hui Ma ${ }^{1, *}$, Ying Lou ${ }^{1}$, Wen Hui Lin ${ }^{1}$, Hong Wei Xue ${ }^{1}$ \\ ${ }^{I}$ National Key Laboratory of Plant Molecular Genetics, Institute of Plant Physiology and Ecology, Shanghai Institutes for Biological \\ Science (SIBS), Chinese Academy of Sciences; and Partner Group of Max-Planck-Institute of Molecular Plant Physiology (MPI-MP) \\ on "Plant Molecular Physiology and Signal Transduction", 300 Fenglin Road, 200032 Shanghai, China
}

Multiple repeats of membrane occupation and recognition nexus (MORN) motifs were detected in plant phosphatidylinositl monophosphate kinase (PIPK), a key enzyme in PI-signaling pathway. Structural analysis indicates that all the MORN motifs (with varied numbers at ranges of 7-9), which shared high homologies to those of animal ones, were located at N-terminus and sequentially arranged, except those of OsPIPK1 and AtPIPK7, in which the last MORN motif was separated others by an $\sim 100$ amino-acid "island" region, revealing the presence of two kinds of MORN arrangements in plant PIPKs. Through employing a yeast-based SMET (sequence of membrane-targeting) system, the MORN motifs were shown being able to target the fusion proteins to cell plasma membrane, which were further confirmed by expression of fused MORN-GFP proteins. Further detailed analysis via deletion studies indicated the MORN motifs in OsPIPK1, together with the 104 amino-acid "island" region are involved in the regulation of differential subcellular localization, i.e. plasma membrane or nucleus, of the fused proteins. Fat Western blot analysis of the recombinant MORN polypeptide, expressed in Escherichia coli, showed that MORN motifs could strongly bind to PA and relatively slightly to PI4P and $\mathrm{PI}(4,5) \mathrm{P}_{2}$. These results provide informative hints on mechanisms of subcellular localization, as well as regulation of substrate binding, of plant PIPKs.

Cell Research (2006) 16:466-478. doi:10.1038/sj.cr.7310058; published online 15 May 2006

Keywords: membrane targeting, PIPK, MORN motif, subcellular localization

\section{Introduction}

The lipid second messenger molecule phosphati-dylinositol 4,5-bisphosphate $\left[\mathrm{PI}(4,5) \mathrm{P}_{2}\right]$ plays critical roles in the regulation of membrane trafficking, cytoskeleton organization, channel activities, nuclear functions, apoptosis, and

Correspondence: Hong Wei Xue

Tel: +86-21-54924059; Fax: +86-21-54924060;

E-mail: hwxue@sibs.ac.cn

*Current address: Unit of Molecular Signal Transduction, ERRB, NICHD, Bldg 49, Rm 6C64, Bethesda, MD 20892

Abbreviations: PI (phosphatidylinositol); PI4P (PI 4-monophosphate); PI5P (PI 5-monophosphate); PI(4,5) $\mathrm{P}_{2}$ (PI 4,5-bisphosphate); PIPK (phosphatidylinositol monophosphate kinase); PA (phosphatidic acid); PAGE (polyacrylamide gel electrophoresis)

Received 8 Feb 2006; revised 1 Mar 2006; accepted 6 Mar 2006; published online 15 May 2006 signal transduction in animal or yeast cells [1-3]. Growing evidence suggests that the synthesis of $\mathrm{PI}(4,5) \mathrm{P}_{2}$ at specific intracellular locations is dynamically regulated, same as the recruitment or the activation of specific proteins that are involved in mediating the distinct downstream effects of $\mathrm{PI}(4,5) \mathrm{P}_{2}$ [4-6]. Moreover, while being itself a critical second messenger, $\mathrm{PI}(4,5) \mathrm{P}_{2}$ also serves as the precursor to other three second messenger molecules, i.e. inositol 1,4,5-trisphosphate $\left(\mathrm{IP}_{3}\right)$, diacylglycerol (DAG), and phosphatidylinositol 3,4,5-trisphosphate $\left[\mathrm{PI}(3,4,5) \mathrm{P}_{3}\right][3,7]$. Consequently, the generation and degradation of $\mathrm{PI}(4,5) \mathrm{P}_{2}$ is also required for initiation of these additional signals. Thus, studies on the mechanisms regulating the turnover of $\mathrm{PI}(4,5) \mathrm{P}_{2}$ is important for understanding many cellular processes regulated by PI signaling.

Phosphatidylinositol phosphate kinases (PIPKs) generate $\mathrm{PI}(4,5) \mathrm{P}_{2}$ and play fundamental role in the regulation of 
cellular processes mediated by $\mathrm{PI}(4,5) \mathrm{P}_{2}$. PIPKs can also phosphorylate other phosphoinositides in vitro and in vivo [8] and thus have the potential to generate multiple lipid second messenger molecules, indicating that PIPKs form a multifaceted family of signaling enzymes distinct from PI 3-kinases and PI 4-kinases. To date, three distinct subfamilies of PIPKs, types I, II, and III, have been identified that share significant sequence homology in the catalytic domains, but differ in the substrate specificities, subcellular localizations, and functions [8]. Type I PIPKs are PI4P 5kinases and phosphorylate PI4P at the D-5 hydroxyl group of inositol ring [9]. Type II PIPKs, in contrast, are PI5P 4-kinases that phosphorylate PI5P at the D-4 position to generate $\mathrm{PI}(4,5) \mathrm{P}_{2}[9]$. The third class, type III PIPKs, of which yeast $\mathrm{Fab} 1$ is the prototype, do not generate $\mathrm{PI}(4,5) \mathrm{P}_{2}$ but rather phosphorylate PI3P to $\mathrm{PI}(3,5) \mathrm{P}_{2}$ [10-12].

The cloning and characterization of the PIPKs serves as an entry point to understand the regulation of PI signaling processes at the molecular level [13, 14]. Moreover, the structure of the first PIPK, PIPK II $\beta$, has been solved [15]. The tertiary structure of PIPK II $\beta$ consists of two identical PIPK II $\beta$ proteins that interact at the $\mathrm{N}$-terminus with each other. The interface of the dimer forms a flat surface area with a net positive charge of +10 , and the charge and the flatness suggest that this region functions as an interface for membrane association via electrostatic interactions [15]. This model provided a possibility for the mechanism for the PIPKs in mammalian cell functionally near the membrane.

The study in plant PI signaling pathway started as early as 1970s, but until 1990s it has made great breakthrough. Now most phosphoinositide molecules presented in mammalian cells have been identified in plant cells, and plant genes encoding the PI metabolism enzymes have been cloned in succession, including those for PI synthase [16], PI 3-kinases [17, 18], PI4K [19], PIPK [20], PI-PLCs [21, 22], inositol polyphosphate phosphatases [23, 24], inositol polyphosphate kinases [25, 26], and others. In 1998, a cDNA of plant PIPK (AtPIP5K1) from Arabidopsis was cloned, which was the first report of plant PIPK [20]. Owing to some structural and biochemical differences, AtPIP5K1 was not categorized as the known subfamily of PIPKs [20]. Since then, some putative PIPKs from Arabidopsis and rice have been predicted and they are more homologous with AtPIP5K1 compared to their homologs in mammalian or yeast cells. PIPKs of Arabidopsis have a conserved domain containing the catalytic core located at the C-terminus, and an unconserved domain close to Nterminus, which harbored varied numbers of novel repeat motifs in different isoforms. These novel repeat motifs were subsequently recognized as MORN (membrane occupation and recognition nexus) motifs. In mammalian cells, the MORN motifs are found and well studied in a protein family of junctional membrane complex proteins, exampled by JP1, and contribute to the plasma membranebinding capacity of JP-1 [27]. Whether the MORN motifs of plant PIPKs play a similar role in the regulation of subcellular localization is unknown yet, but would be a plausible hypothesis.

Up to now, little knowledge about the subcellular localizations of the key enzymes involved in PI pathway has been obtained. Several enzymes such as PI synthase and PLC have been demonstrated to be membrane proteins [16, 22]. However, some others, including PIPKs, could not be confirmed to be membrane locating based on the physical characteristics of the peptides, although there are some clues that the enzymes are functioned near the cell membranes. Studies on the functions of the specific regions presented in PIPKs to detect whether they are related to membrane location will certainly provide hints for the functional mechanism, as well as the biochemical characteristics, of PI signaling pathway.

We have previously cloned a rice PIPK cDNA encoding OsPIPK 1 and found that there are eight MORN repeat motifs exiting in the N-terminus [28]. Further similarity matrix analysis revealed an additional MORN motif. In this study, with the help of the modified yeast sequence of membrane-targeting [SMET, 29] system, we are able to show that the multiple MORN motifs presented in PIPKs also contribute to membrane targeting and that different numbers of MORN motifs showed differential membranetargeting capacities. Further studies employing GFP fusion proteins revealed the differential subcellular localizations of truncated MORN motifs. In addition, Fat Western blot analysis of the recombinant MORN region polypeptide containing the whole nine MORN motifs and the linker sequence showed that it could bind phosphatidic acid (PA), PI4P, and $\mathrm{PI}(4,5) \mathrm{P}_{2}$, providing hints on regulatory mechanisms of plant PIPKs.

\section{Materials and methods}

\section{Enzymes and chemicals}

Enzymes used for DNA restriction and modification were obtained from Boehringer (Mannheim, Germany). DNA primers for polymerase chain reaction (PCR) and Taq polymerase were from Genecore (Shanghai). Yeast nitrogen base (YNB) without amino acids was obtained from DIFCO Co. (Catalog no. 291940). All amino acids used were from Sigma (Missouri, USA). All inositol phosphates are named as the D-myo isomer. Synthetic dipalmitoyl phosphatidylinositol 5-monophosphate (PI5P, P1168), phosphatidylinositol 4-monophosphate (PI4P, P7686), and phosphatidylinositol 4,5-bisphosphate [PI(4,5) $\left.\mathrm{P}_{2}, \mathrm{P} 8707\right]$, as well as phosphatidic acid (PA, P4013), phosphatidylinositol (PI, P8443), and phosphatidylcholine (PC, P7443), were purchased from Sigma (Missouri, USA). 
Bacteria, yeast, and plants

Escherichia coli XL1-blue cells were used to amplify the construct and to perform the relevant subcloning. Cells were cultivated at 37 ${ }^{\circ} \mathrm{C}$ in LB medium supplemented with appropriate antibiotics using standard methods [30]. Saccharomyces cerevisiae temperaturesensitive mutant strain cdc25 H (Stratagene Inc) was used for yeast transformation and further growth. Yeast cells were cultivated at 25 ${ }^{\circ} \mathrm{C}$ or $37^{\circ} \mathrm{C}$ in YPAD medium or Synthetic Glucose Minimal Medium (SD medium) with appropriate $10 \times$ Dropout Solution, following the protocol provided by the Stratagene. Seeds of rice (Oryza sativa L. $\mathrm{cv}$. Zhonghua 11) were germinated on agar-solid MS medium, and then grown in water in a phytotron with a 12 -h light $\left(26^{\circ} \mathrm{C}\right)$ and 12 -h dark $\left(18^{\circ} \mathrm{C}\right)$ period. Two-week-old plants grown in water were used for RNA extraction.

\section{Analysis of PIPK structural organizations}

Structural analysis of the protein sequence was performed on the ExPASy Molecular Biology Server (http://cn.expasy.org/) or using the SMART sequence analysis program.

Constructs containing the MORN motifs for SMET assays

The primers used to construct the vector containing the OsPIPK1 MORN motifs for SMET analysis are listed in Table 1 and shown in Figure 1. Primers OsPIPK1-9-F and OsPIPK1-9-R were used for the construction of pSos-OsPIPK1, which contains the whole 9 MORN motifs and the 104-amino-acid linker. Primers OsPIPK1-6-F, OsPIPK1-5-F, OsPIPK1-4-F, and OsPIPK1-2-F with the OsPIPK1-9-R were used for the construction of pSos-OsPIPK1-6, pSos-OsPIPK1-5, pSos-OsPIPK1-4, and pSos-OsPIPK1-2, respectively (containing 6, 5,4 or 2 MORN motifs from the C-terminus, Figure 1A). Primers MORN-F and MORN-R, DeMORN-F and DeMORN-R were used for construction of pSos-MORN (only containing one MORN motif) and pSos-DeMORN (containing the 100-amino-acid linker and the last MORN motif), respectively. For these constructions, DNA fragments were amplified by PCR, digested with $\mathrm{BamH} \mathrm{I} / \mathrm{Sal}$ I, and subcloned into pSos vectors precut with the same enzymes.

Table 1 Sequences of primers used for constructs of SMET system and GFP fusion studies. Restriction enzyme sites are underlined

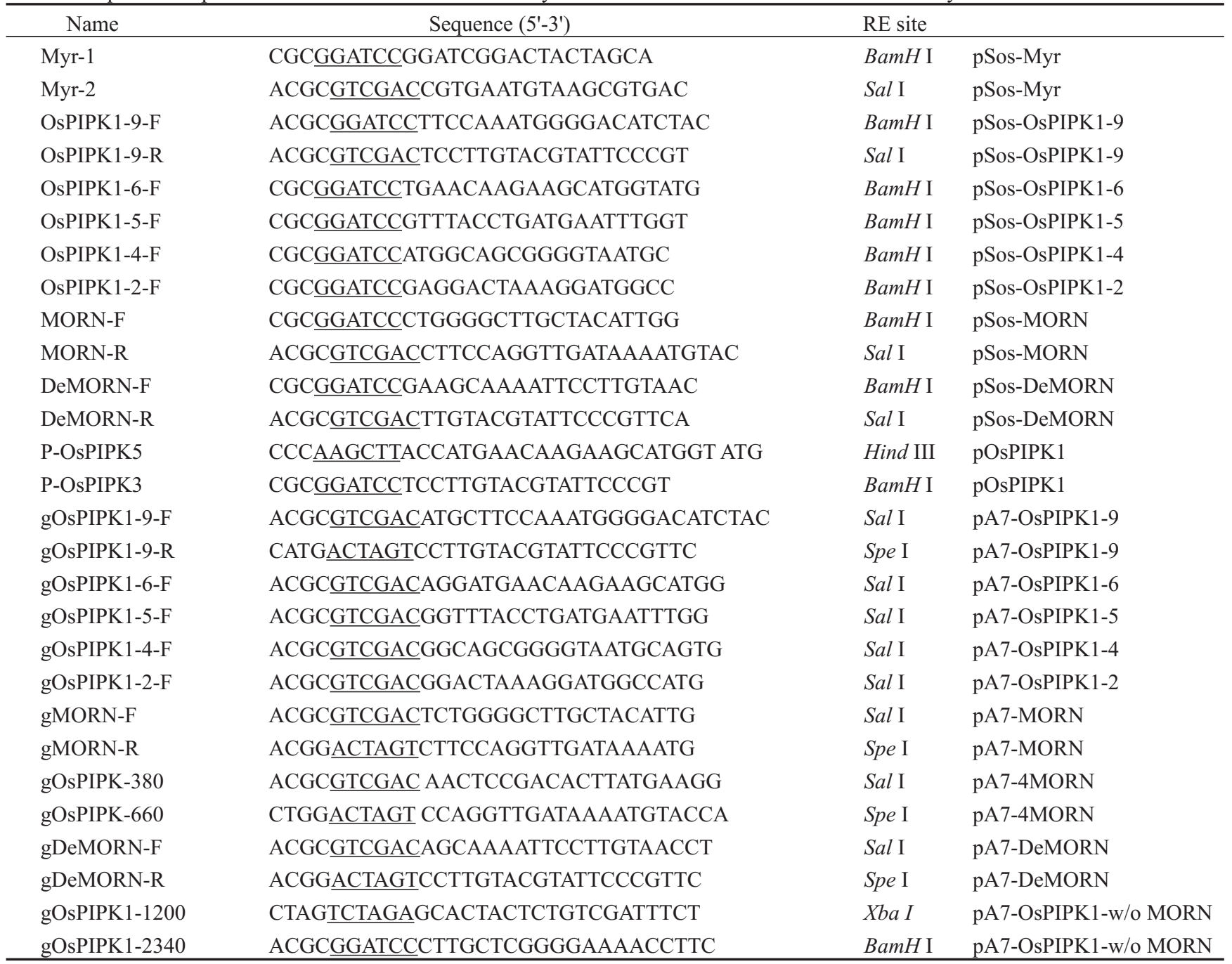






Figure 1 SMET analysis indicates the membrane-targeting capabilities of MORN motifs. (A) Positions of the primers used for construction of the vectors containing the MORN motifs of OsPIPK1 for SMET studies. Sequences are listed in Table 1. (B) Yeast cdc $251 \mathrm{H}$ cells transformed with pSos-Myr, the positive control, could grow at both the permissive $25^{\circ} \mathrm{C}$ and the restrictive $37^{\circ} \mathrm{C}$, while transformants containing pOsPIPK 1 and pSos, used as the negative controls, could grow well at $25^{\circ} \mathrm{C}$ but not at $37^{\circ} \mathrm{C}$. Those transformed with pSos-OsPIPK1-9, pSos-OsPIPK1-6, pSos-OsPIPK1-5, pSos-OsPIPK1-4, pSos-OsPIPK1-2, or pSos-DeMRON could also grow at both $25^{\circ} \mathrm{C}$ and $37^{\circ} \mathrm{C}$, while transformed with pSos-MRON could not grow at $37^{\circ} \mathrm{C}$. Results were summarized in the last two figures. The yeast cells were transformed with these vectors of 1 , pSos-Myr; 2, pSos; 3, pOsPIPK1; 4, pSos-OsPIPK16; 5, pSos-OsPIPK1-5; 6, pSos-OsPIPK1-4; 7, pSos-OsPIPK1-2; 8, pSos-DeMORN; 9, pSos-MORN. (C) Comparison of relative membrane-targeting capacities of MRON motifs. Ratios were calculated through comparing the growing colonies at $37^{\circ} \mathrm{C}$ to those at $25^{\circ} \mathrm{C}$. Ratio of positive control pSos-Myr was regarded as $100 \%$, which allows the quantitative comparison of membrane-targeting abilities between different truncated forms of MORN motifs. 
DNA fragments amplified with primers P-OsPIPK-F and POsPIPK-R were used for the construction of pOsPIPK1, resulting in constructs deficient in the hSos-coding sequence, which served as independent negative control. The amplified DNA fragment was digested with Hind III/BamH I and subcloned into pSos vectors previously digested with the same enzymes, resulting in the deletion of the hSos-coding sequence.

SMET assay was performed as described [29]. In short, yeast cdc $25 \mathrm{H}$ cells were transformed with appropriate vectors (vectors pSos and pSos-Myr, which served as the negative and positive controls, respectively), and incubated on selective medium (synthetic medium lacking Leu) at $25{ }^{\circ} \mathrm{C}$ for $48 \mathrm{~h}$ and then the transformants were replica plated onto the same selective medium and were incubated at $37^{\circ} \mathrm{C}$ for another $48 \mathrm{~h}$. Cell growth were observed and number of independent colonies were then calculated. All transforma- tion and yeast cultivation procedures were according to Stratagene's instruction manual (\#200444).

\section{Fusion expression of MORN motifs with GFP}

To study the detailed localization of MORN motifs in vivo, fusion expression of different numbers of MORN motifs with GFP were performed. Plasmid pA7-GFP, in which the CaMV 35S promoter is located upstream of $g f p$, was kindly provided by Dr K Czempinski, University of Potsdam, Germany, and used for generation of fusion proteins combining GFP with different numbers of MORN motifs with or without the 104-amino-acids linker. DNA fragments encoding different numbers of MORN motifs were PCR amplified (Table 1 and Figure 2A). Primers gOsPIPK1-9-F and gOsPIPK1-9-R were used for the construction of pA7-OsPIPK1-9, which harbored all of

A

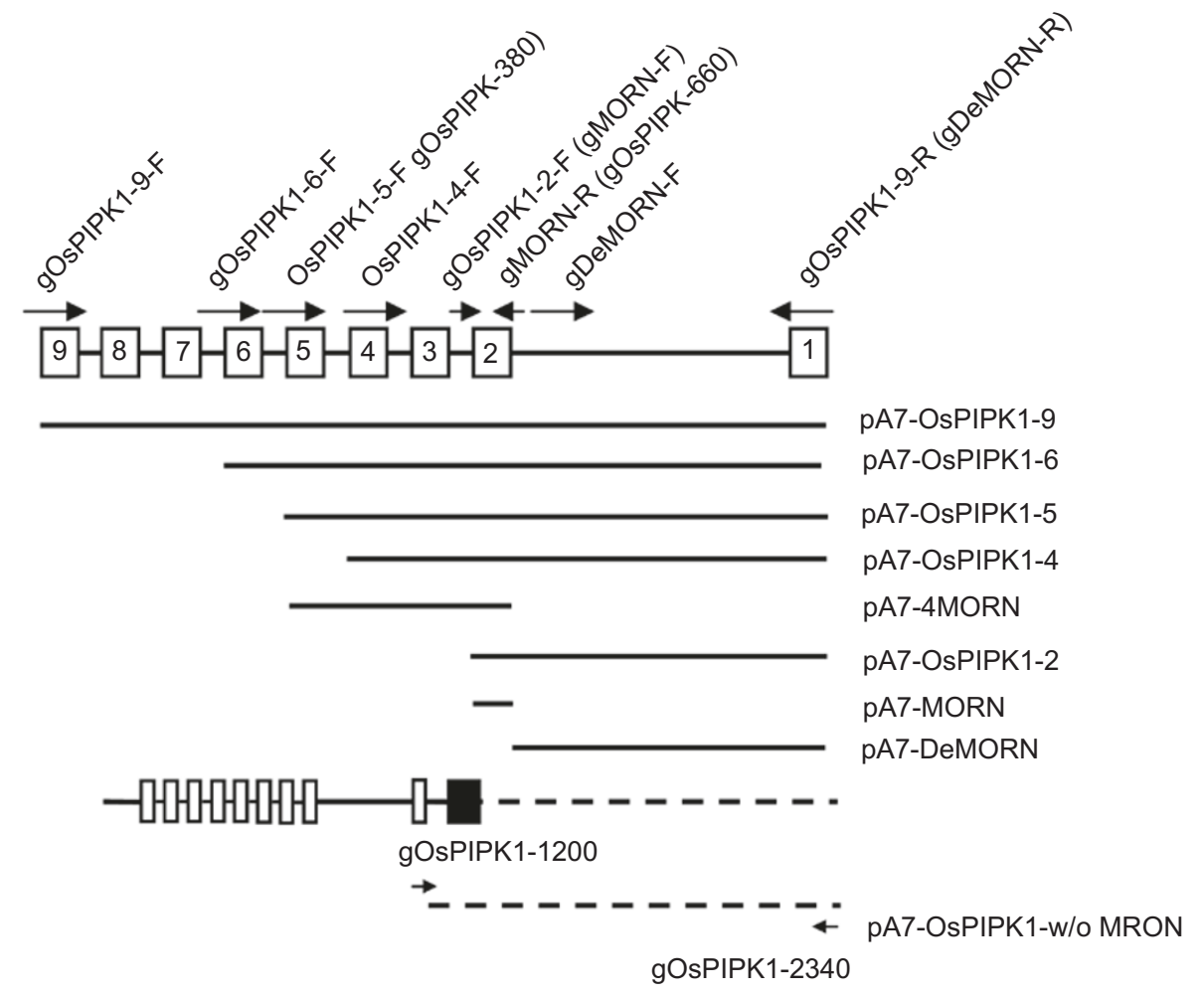

Dimerization region

Figure 2 Transient expression of GFP fusion protein with different numbers of MORN motifs in onion epidermal cells indicated the involvement of MORN motifs in the regulation of differential subcellular locations. (A) Positions of the primers used for construction of the vectors containing the MORN motifs of OsPIPK1 for GFP fusion studies. Sequences are listed in Table 1. (B) Expression of fused GFP protein in onion epidermal cells. Detection of green fluorescences of fused GFP with the whole MORN region of OsPIPK1 (pA7-OsPIPK1-9), as well as the N-terminus deletions (pA7-OsPIPK1-6; pA7-OsPIPK1-5; pA7-OsPIPK1-4; and pA7-OsPIPK12) indicated plasma membrane and/or nucleus localizations after plasmolysis. The fusion protein containing the last MORN motif with the linker mostly concentrates in the nucleus. And the fusion protein with only one MORN motif is detected through the whole cell. The empty vector pA7-GFP was used as control. Fused AMT2 was used as the positive control. Cell plasmolysis was achieved by incubating onion epidermal strips in $1 \mathrm{M}$ sucrose for $10 \mathrm{~min}$. The images obtained by the observation with a confocal scanning microscope were shown in a gallery series (right panel), and the representative images were shown in the left panel. (C) Truncated mutant of OsPIPK1 lacking MORN motifs showed irregular localization throughout the cells, indicating the crucial roles of MORN motifs in the membrane localization of the proteins. 
B

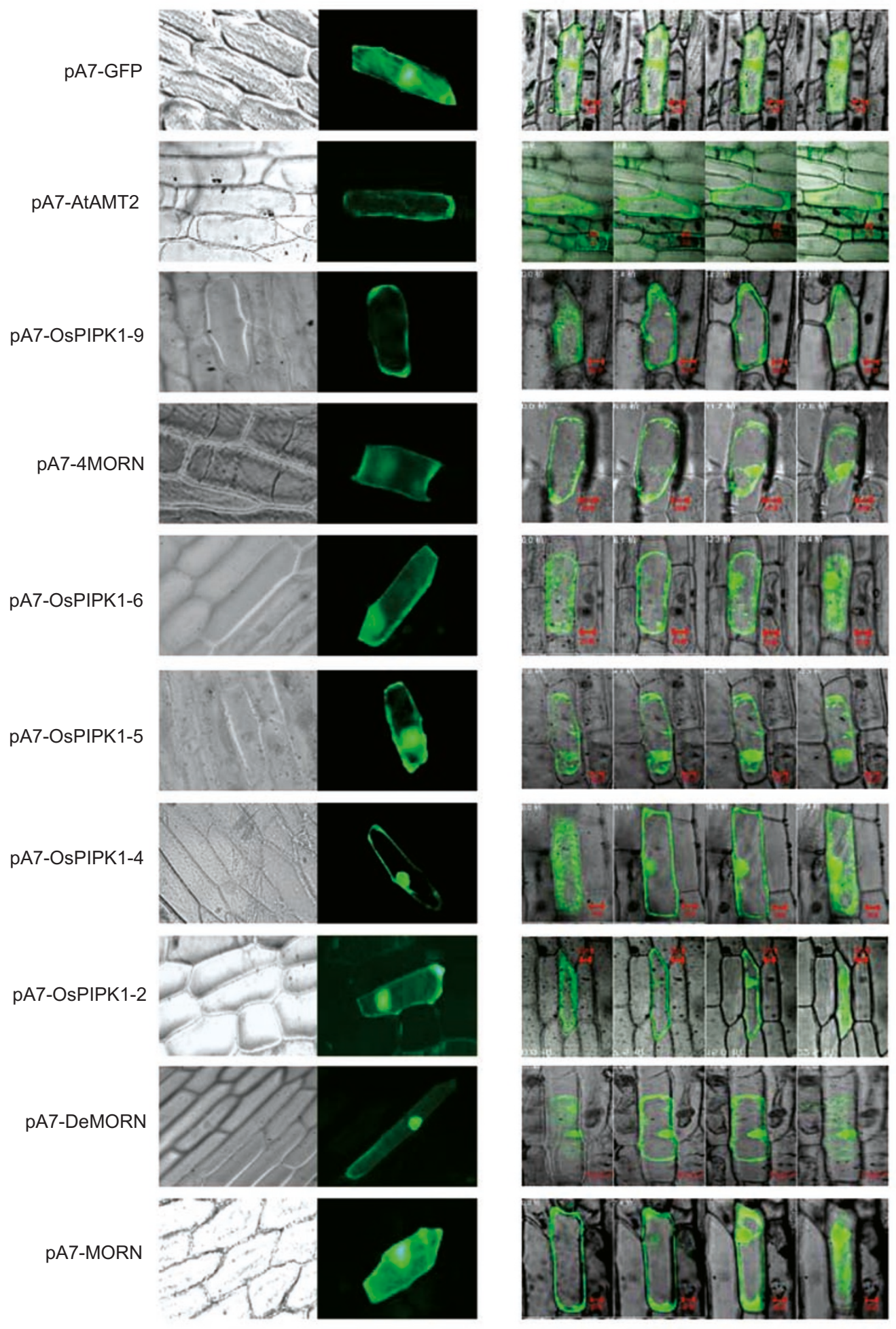

C

pA7-OsPIPK1-w/oMORN 
9 MORN motifs and the linker. Primers gOsPIPK-6-F, gOsPIPK-5-F, gOsPIPK-4-F, or gOsPIPK-2-F with gOsPIPK1-9-R were used for the construction of pA7-OsPIPK1-6, pA7-OsPIPK1-5, pA7-OsPIPK14, or pA7-OsPIPK1-2, respectively, which harbored 6, 5, 4 or 2 MORN motifs with the linker. Primer gMORN-F with gMORN-R or gDeMORN-F with gDeMORN-R was used for the construction of pA7-MORN or pA7-DeMORN, respectively, resulting in the fusion of GFP with single MORN motif or the linker plus the last MORN motif. Primers gOsPIPK-380 and gOsPIPK-660 were used for the construct of pA7-4MORN, which contains 4 MORN motifs at positions 2-5. Primers gOsPIPK1-1200 and gOsPIPK1-2340 were used for the construct of pA7-OsPIPK1-w/o MORN, resulting in the fusion of GFP with OSPIPK1 deletion of MORN motifs. PCR-amplified DNA fragments were digested with the appropriate enzymes and subcloned into pA7-GFP vectors previously digested with the same enzymes. All constructs were sequenced to confirm in-frame reading.

\section{Particle bombardment and GFP fluorescence detection}

Transient expression of GFP-fusion proteins in onion epidermal cells was performed with a Model PDS-1000/He Biolistic Particle Delivery System (BioRad, CA, USA). Five micrograms of purified plasmids were coated onto $0.6-1 \mu \mathrm{m}$ gold particles as described by the manufacturer. Bombardment was performed as the following parameters: 1100 psi rupture disc; $27 \mathrm{in.} \mathrm{Hg}$ vacuum; and $6 \mathrm{~cm}$ distance from the stopping screen to the target tissues. After bombardment, onion pieces were incubated on solid MS medium at $25{ }^{\circ} \mathrm{C}$ under darkness for at least $24 \mathrm{~h}$. Cell plasmolysis was performed through incubation of onion pieces in $1 \mathrm{M}$ sucrose solution for $10 \mathrm{~min}$.

GFP-expressing cells were detected using a florescence microscope (Leica DMR) equipped with a GFP filter (band pass range is $470-525 \mathrm{~nm}$ ) to mask the red fluorescence of chlorophyll, thereby permitting visualization of the green fluorescent GFP-expressing cells. An imaging system (Leica DC300F) was attached to the fluorescence microscope to capture the image in real time using Leica Qwin Plus software. In addition, green fluorescence was further detected with a Confocal Laser Scanning Microscope (Zeiss LSM 510 META, excitation wavelength $488 \mathrm{~nm}$ by argon laser, emission wavelength $505-530 \mathrm{~nm}$ by BP filter), and section Z-series of images were collected at different interval through the specimens to facilitate the observation. Twenty to thirty cells were imaged for each experiment. Empty vector pA7-GFP and construct pAt-AtAMT2, harboring GFP-AtAMT2 fusion (AtAMT2 encodes a high-affinity ammonium transporter located at the plasma membrane, 31), were used as negative and positive controls, respectively.

\section{Recombinant expression and purification of OSPIPK1 MORN region}

The cDNA fragment encoding the OsPIPK1 MORN region was amplified by PCR with primers that flanked either end of the region, i.e. DB-OsPIPK1-5 (5'-CCG GAA TTC ATG AAC AAG AAG CAT GGT ATG-3', added EcoR I site underlined), and DB-OsPIPK1-3 (5' -ACC GCT CGA GTC CTT GTA CGT ATT CCC GT-3', added Hind III site underlined), and then subcloned into the expression vector pET-32a (Novagen, USA). Rosetta cells were transformed with the recombinant construct, and expression of the recombinant peptide was induced with the addition of isopropyl- $\beta$-D-thiogalactopyranoside ( $1 \mathrm{mM}$ final concentration) to the cell culture. After incubation of $0.5,1,2,3$ and $5 \mathrm{~h}$, the cells were collected and lysed by sonica- tion, solubilized in lysis buffer with $8 \mathrm{M}$ urea, and purified by metal affinity chromatography using Ni-NTA Spin columns according to the protocol by the manufacturer (Qiagen, USA). Because the peptide was insoluble, purification was carried out under denaturing conditions with solutions containing $8 \mathrm{M}$ urea and column fractions were then dialyzed with native condition (solution containing imidazole according to the manufacturer protocol) to remove urea and to promote refolding. Both the crude extract and purified peptide were used for SDS-PAGE electrophoresis.

\section{Fat Western blotting}

Binding capacity of MORN region with different phospholipid molecules were performed by Fat Western blotting according to Stevenson [32]. In detail, the phospholipids were solubilized in chloroform as stock solutions of $1 \mathrm{mg} / \mathrm{ml}$, and a minimum of 10 $\mu \mathrm{l}$ containing $0.5,1.0$ or $5.0 \mu \mathrm{g}$ of lipid were spotted onto nitrocellulose (NitroBind, MSI, USA). The dotted membrane was dried at $24{ }^{\circ} \mathrm{C}$ for $1 \mathrm{~h}$ and the nitrocellulose was then incubated with $3 \%$ (w/v) fatty acid-free bovine serum albumin (A-6003, isolated by cold ethanol precipitation; Sigma) in TBST [10 mM Tris ( $\mathrm{pH} 8.0$ ), $140 \mathrm{mM} \mathrm{NaCl}$, and $0.1 \%(\mathrm{v} / \mathrm{v})$ Tween 20] for $1 \mathrm{~h}$ and then placed in a solution containing the His-tagged fusion proteins comprising the whole MORN region (9 MORN motifs and the linker) at $4{ }^{\circ} \mathrm{C}$ overnight with shaking. The membrane was washed with TBST three times for 10 min each and then incubated with His-Tag Monoclonal Antibody (Novagen, USA) to the His-tagged region diluted at 1:1 000 in TBST for $1 \mathrm{~h}$ at $24^{\circ} \mathrm{C}$. The incubated membrane was then washed three times for $10 \mathrm{~min}$ in TBST at $24^{\circ} \mathrm{C}$ and then incubated with Goat Anti-Mouse IgG AP Conjugate (Novagen, USA) at a titer of 1:5000 in TBST for $1 \mathrm{~h}$ at $24^{\circ} \mathrm{C}$. The membrane was washed again in TBST three times for $10 \mathrm{~min}$ and then incubated for $10 \mathrm{~min}$ in the $1 \times$ AP developing solutions containing 1:1 mixture of NBT and BCIP (Novagen, USA) for subsequent colorimetric detection.

\section{Results}

Presence of multiple MORN motifs in OSPIPK1, as well as other plant PIPKS

MORN motif containing a conserved peptide with 14 amino acids in length (Y-Q/E-G-E/Q-T-X-N-G-K-X-H-G$\mathrm{Y}-\mathrm{G})$, is a novel protein-folding module shared by functionally different proteins with unclear physiological roles. We have reported previously the presence of 8 MORN motifs in rice PIP 5-kinase, OsPIPK1, of which the MORN motifs localized in the N-terminus at positions 41-215 [28]. However, further similarity matrix analysis revealed an additional MORN motif localized at 320-333, with a 104 amino-acids linker sequence to the former MORN motifs. This new MORN (YEREYVQGVLIMEQ), similar to the second MORN motif in the Caenorhabditis elegans JP homolog [27], shared common characteristic of MORN motifs, especially in its anterior sequence.

To elucidate the distribution of MORN motifs in plant PIPKs, rice genome was searched, and of 8 OsPIPKs at present, including OsPIPK1, 5 rice PIPKs contain the MORN motifs. Of the other three putative OsPIPKs, two 
A

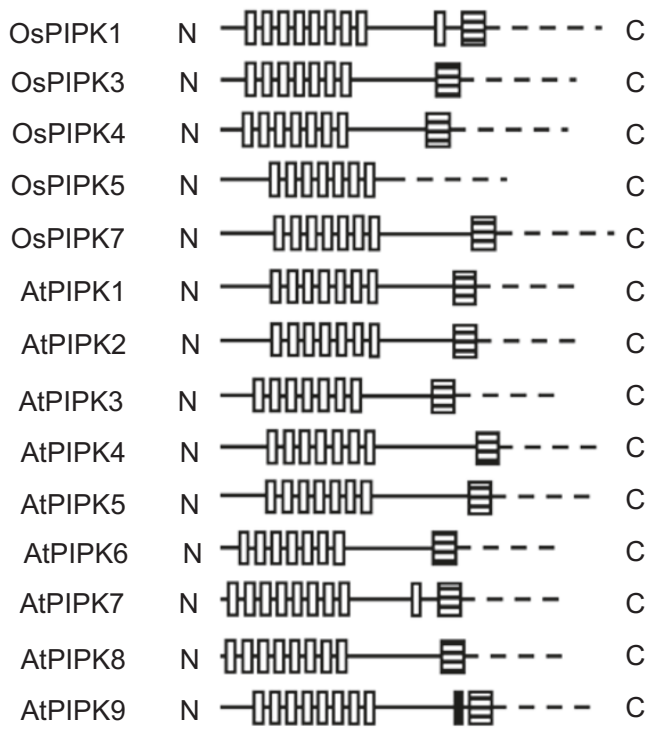

100 aa [ MORN motif 目Dimerization region

NLS

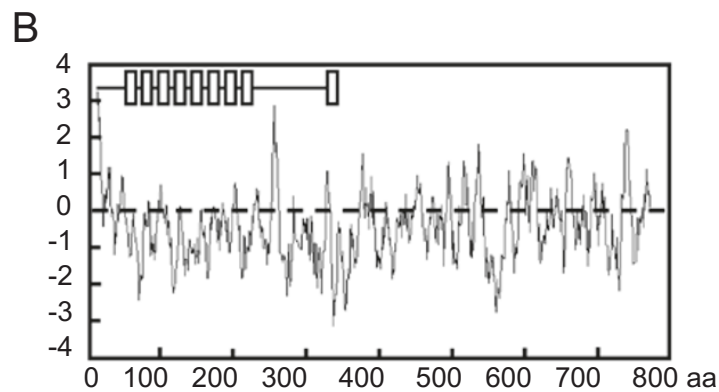

Figure 3 Presence of multiple MORN motifs in plant PIPKs. (A) Presence of multiple MORN motifs in Arabidopsis and rice PIPKs. MRON motifs are presented at N-terminus and repeated for 7-9 times. There are two types of MORN structure in plant PIPKs, represented by OsPIPK1 and AtPIPK7. Note that only AtPIPK9 has nuclear localization signal (NLS). (B) Hydropathy plots deduced from the OsPIPK1 amino-acid sequences were generated with the Kyte-Doolittle algorithm [37], where increased hydrophobicity is denoted by positive values. Amino-acid residues 41-333 of OsPIPK1 are MORN motif domain. The positions for MORN motifs and the linker sequences are indicated by box and line, respectively.

of them, OsPIPK2 and OsPIPK8, are truncated peptides that lacked the N-terminus and the other, OsPIPK6, lacks the corresponding EST sequence in the database. In another model plant, Arabidopsis, there are 11 genes encoding PIPK proteins, which are highly similar to AtPIP5K1 [33], and only two proteins lack the MORN motifs. However, whether these two putative AtPIPKs are expressed is at present unclear, as no mRNA or EST sequences have been reported for either of them [33]. Structural organization




analysis showed that the numbers and positions of the MORN motif repeats in Arabidopsis and rice PIPKs are slightly different (Table 2, Figure 3A).

Further hydropathicity analysis showed that there is no putative transmembrane segment existing in the MORN region of OsPIPK1 (Figure 3B), including the linker sequence. More importantly, the MORN motifs are full of negative charge (Figure 3B); however, the physiological meaning of which is unclear. Interestingly, the MORN motifs are only found in the plant PIPKs, but not yet in the mammalian or yeast PIPKs. Although the physiological function of the MORN motif is not clear, there was a clue that hinted the MORN motifs may be involved in the membrane localization of plant PIPKs [27].

\section{Structural analysis revealed two kinds of MORN motif arrangements}

In OsPIPK1, the anterior 8 MORN motifs are linked closely, whereas the last one is not, with a linker sequence of 104 amino acids in length between them. After analyzing the structures of MORN motifs in other OsPIPKs and AtPIPKs, we found that the AtPIPK7 also has one similar linker, which is 90 -aa long. Other than these two PIPKs, the other PIPKs in Arabidopsis and rice have no such kind of linker sequence and their MORN motifs are arranged closely, suggesting the presence of two kinds of MORN motif arrangements in plant PIPKs. In addition, no subcellular signal sequence or special structural characteristic was found in the linker sequence.

\section{SMET analysis indicates membrane-targeting ability of the MORN motifs}

Yeast-based SMET system was employed to detect whether MORN motifs has the capability of membrane targeting. The SMET system is based on the modification of Cyto Trap two-hybrid system (Stratagene Inc.) and it exploits the application that expression of human SOS protein and its subsequent translocalization to plasma membrane will activate the RAS pathway and thus will keep yeast strain cdc25H viable at $37^{\circ} \mathrm{C}$ (for details, see [29]). To exclude the possibility that the MORN motifs themselves could stimulate the RAS pathway, pOsPIPK1 was used as negative control, which was constructed through deletion of the hSos sequences originally presented in the pSos vector to yield construct encoding only the MORN region (9 MORN motifs plus the linker sequence). Transformed yeast cells grew normally at $25^{\circ} \mathrm{C}$ but not at $37^{\circ} \mathrm{C}$ (Figure 1B), demonstrating that the whole MORN region itself could not activate the RAS pathway.

Next, we examined whether the whole MORN region was capable of membrane targeting. As shown in Figure 1B, yeast cdc25H cells transformed with pSos-OsPIPK1-9 grew


Figure 4 Recombinant expression, purification, and Western blot analysis of the OsPIPK1 MORN region. (A) SDS-PAGE analysis of $E$. coli crude protein extracts after induction with IPTG (1 mM final concentration) for $0.5,1,2,3$, or $5 \mathrm{~h}$. Arrow shows the position of induced recombinant proteins. (B) SDS-PAGE analysis (left) or Western blot analysis of purified recombinant MORN peptide (fractions 1 and 2 eluted from the immunoaffinity column) using His-Tag monoclonal antibody. The arrow indicates the protein band with the expected molecular mass of $45 \mathrm{kDa}(25 \mathrm{kDa}$ of predicted MW of the MORN peptide and $20 \mathrm{kDa}$ of the insert sequence in the pET-32a vector).

well at the restrictive temperature of $37^{\circ} \mathrm{C}$, suggesting the MORN region itself was capable of membrane targeting. As expected, yeast cells containing pSos-Myr, serving as positive control, grew well at $37^{\circ} \mathrm{C}$, whereas those containing pSos and pOsPIPK1, two negative controls, showed no cell growth at $37^{\circ} \mathrm{C}$.

Further, a serial of deletion within the region of MORN motifs were performed and the SMET analysis of different truncated MORN region (pSos-OsPIPK1-6, pSosOsPIPK1-5, pSos-OsPIPK1-4, pSos-OsPIPK1-2) showed the normal growth of the yeast cells at $37^{\circ} \mathrm{C}$ (Figure 1B), although with different growth velocities, suggesting that at least the last two MORN motifs with the linker are capable of targeting peptides to the membrane. Interestingly, yeast cells containing pSos-DeMORN showed weak growth at $37^{\circ} \mathrm{C}$, indicating that the peptide comprising of the linker 
and the last MORN motif has somewhat membrane-binding ability. However only one copy of MORN motif (pSos-MORN) resulted in lost viability of yeast cells at 37 ${ }^{\circ} \mathrm{C}$, suggesting that only one copy of MORN motif is not enough for the membrane binding and that the linker with the last MORN motif may have their own contribution to the membrane binding.

Yeast colonies growing at $25^{\circ} \mathrm{C}$ and $37^{\circ} \mathrm{C}$ were further

A
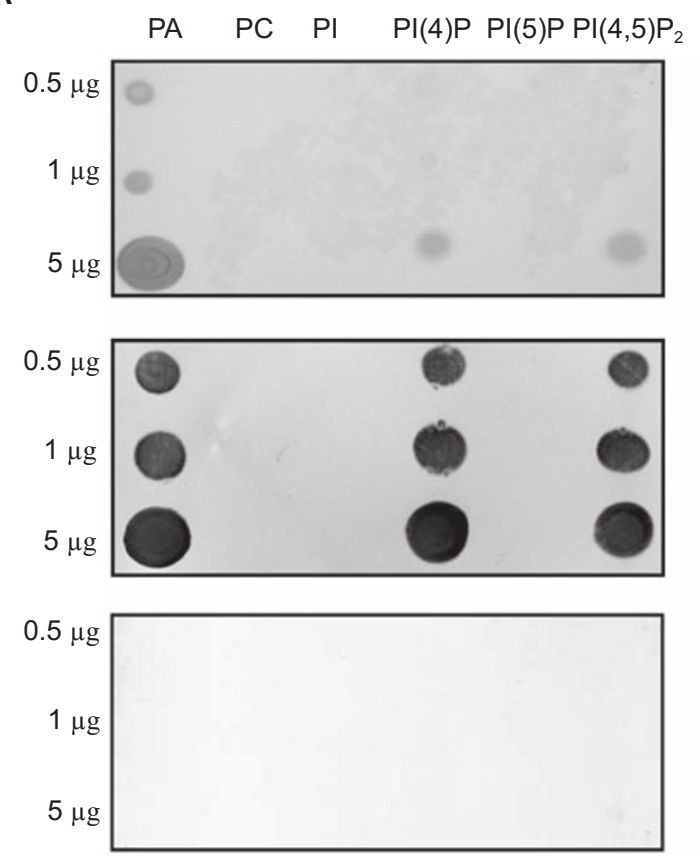

B

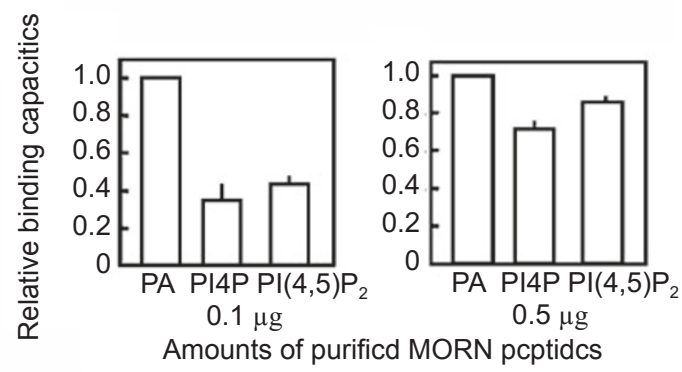

Figure $5 \mathrm{MORN}$ region could bind $\mathrm{PA}, \mathrm{PI} 4 \mathrm{P}$, and $\mathrm{PI}(4,5) \mathrm{P}_{2}$ in vitro. (A) Tested phospholipid molecules are indicated above each blot, and different amounts of lipids $(0.5,1$ or $5 \mu \mathrm{g})$ were spotted onto the nitrocellulose membrane. The blot was incubated with purified recombinant MORN polypeptide (with fused His-tag; $0.1 \mu \mathrm{g} / \mathrm{ml}$, upper panel or $0.5 \mu \mathrm{g} / \mathrm{ml}$, middle panel). Purified peptides from $E$. coli cells transformed with empty pET32a vector were used as negative control (lower panel). (B) Colorimetric analysis of affinity of MORN region with $\mathrm{PA}, \mathrm{PI} 4 \mathrm{P}$, and $\mathrm{PI}(4,5) \mathrm{P}_{2}$. The concentration of peptide is $0.1 \mu \mathrm{g} / \mathrm{ml}$ (left panel) or $0.5 \mu \mathrm{g} / \mathrm{ml}$ (right panel). counted to quantitate the membrane-targeting capabilities of the various truncated MORN region. As shown in Figure $1 \mathrm{C}$, the whole MORN region showed $103 \%$ targeting capability comparing to that of the positive control (pSosMyr), suggesting that entire MORN region has strong membrane-targeting capability as that of Myr sequence, which is regarded as the plasma membrane-localized signal. Sequential deleting of MORN motifs resulted in the decreased targeting capability. The targeting capability of peptides with 6, 5, 4 and 2 copies of MORN motifs showed $98 \%, 91 \%, 84 \%$, and $81 \%$ of the targeting capability as compared to the positive control, respectively. Surprisingly, when deleted one copy of MORN motif from the pSos-OsPIPK1-2, the targeting capability of remaining linker and last one MORN motif sequence sharply reduced to $25 \%$, suggesting that MORN motif is necessary for the membrane binding of the whole peptide and that the interaction of MORN motif with the linker sequence may be important for the membrane binding. This result was consistent with the morphology of yeast cells containing pSos-DeMORN described above. Only one MORN motif showed almost no membrane-targeting abilities, similar to that of the negative controls, pSos and pOsPIPK1 (Figure $1 \mathrm{~B}$ and $1 \mathrm{C})$.

GFP fusion studies revealed the membrane-targeting capacities of MORN motifs, and further the involvement of regulation on differential subcellular localizations

In order to confirm the results by the SMET analysis, and furthermore, to investigate the possibility that peptides bearing different number of MORN motifs would have different subcellular localization, OsPIPK1-green fluorescent protein (GFP) fusion proteins were constructed (Figure 2A) and the results showed that the fusion protein carrying the whole MORN region (pA7-OsPIPK1-9) was localized at the plasma membrane (Figure 2B), suggesting that the MORN motifs itself indeed harbor the ability of membrane targeting.

A serial of deletion of MORN motifs were performed and the analysis showed the fluorescence were detected on the membrane when the fusion proteins carrying 6,5 , 4 , and 2 MORN motifs, however, the fluorescences were also detected in the nucleus and cytoplasm (Figure 2B). Furthermore, pA7-DeMORN bearing the linker and the last MORN motif was detected predominantly in nucleus, and pA7-MORN bearing a single copy of the motif was expressed mainly in the cytoplasm.

Further analysis showed that the truncated mutant of OsPIPK1 lacking MORN motifs showed irregular localization throughout the cells (Figure 2C), indicating that without the MORN motifs, OsPIPK1 would lose the capability to sublocalize correctly. The proposed interaction of OsPIPK1 
with the target component(s) on the membrane or in the nucleus would most likely depend upon the number and/or sequence of MORN motifs.

OsPIPK1 MORN region could bind phosphatidic acid (PA), $P I 4 P$, and $P I(4,5) P_{2}$ in vitro

To elucidate the interacting mechanism of MORN motifs with membrane, we tested the hypothesis that MORN motif would target the membrane through a peptide-lipid binding method. The whole MORN region of OsPIPK1 was recombinantly expressed as a fusion protein with a polyhistidine tag in E. coli Rosetta cell line. The recombinant polypeptide was purified by affinity chromatography (Figure 4) and was eluted under the native condition to restore the natural folding structure. The binding capacity of OsPIPK1 MORN region to different phospholipid molecules was studied via Fat Western blotting, a developed lipid-protein binding assay [32]. As Figure 5 shows, the MORN region could strongly bind to PA, and slightly bind to PI4P and $\mathrm{PI}(4,5) \mathrm{P}_{2}$, but not phosphatidylcholine, PI, or PI5P (Figure 5A). Next, the colorimetric analysis by using the GIS software (Tanon Co. Shanghai) showed that in a lower concentration $(0.1 \mu \mathrm{g} / \mathrm{ml})$, MORN region bound to PI4P and PI $(4,5) \mathrm{P}_{2}$ with similar affinity, but that to PA was more prominent (Figure 5A, upper panel; Figure $5 \mathrm{~B}$, left panel), suggesting that MORN region in OsPIPK1 naturally has the preference to bind to PA. However, when increased the peptide concentration to $0.5 \mu \mathrm{g} / \mathrm{ml}$, MORN region bound to all the three lipids with similar affinity (Figure 5A, middle panel; Figure 5B, right panel).

\section{Discussions}

In this study, we demonstrated the presence of MORN motifs in plant PIPK proteins, and by employing the yeast SMET system and studying the GFP fusion protein expression, we showed that MORN region have the ability of membrane binding, and furthermore, different numbers of MORN motifs result in the differential subcellular locations. These raised the possibility that MORN region is involved in regulating the localization of OsPIPK1. Together with the fact that the PIPKs sharing MORN motifs prevail in Arabidopsis and rice, this further implied that the MORN motifs, together with the binding partner(s) on the membrane or other compounds, is critical for the subcellular location of plant PIPKs.

The fact that mammalian or yeast PIPKs lacked the MORN motifs indicates the great difference in the mechanism of membrane docking between plant PIPKs and mammalian or yeast PIPKs. In mammalian cells, the reported tertiary structure of human PIPK II $\alpha$ consists of two identical II $\alpha$ proteins that interact at the $\mathrm{N}$-terminus
[15]. In addition, it had been demonstrated that the dimerlized protein is anchored on the membrane bilayer and only electrostatic interactions between the protein and the phospholipid are involved in docking. Plant PIPKs that contain the MORN motifs are usually larger than animal or yeast PIPKs, thus raising the following questions: is one unit of plant PIPK enough for the membrane binding, or is the membrane binding of plant PIPKs involved in the interaction of protein factor, or, just like the PIPK II $\alpha$, only the phospholipid interaction are involved? These questions need further investigations.

In mammalian cells, the best characterized protein containing MORN motifs is JP1 (Junctophilin 1), which belongs to a family of junctional membrane complex protein [27]. The MORN motifs repeated 8 times in the N-terminus of JP1 and divided into two groups: the first 6 motifs are one group, the last 2 motifs are the other group, and a 50 -amino-acid linker is between these two groups. GFP fusion protein studies revealed that MORN motifs could help JP1 localize in different subcellular localizations. For example, the fusion peptide carrying all the MORN motifs was localized at the membrane, and those lacking the $\mathrm{N}$ terminal 2 or 4 copies of the motifs were also detected on the membrane. However, the fusion peptide bearing the $\mathrm{N}$-terminal 6 copies of the motifs was detected predominantly in nucleus, and the one carrying the C-terminal 2 copies was expressed on the membrane and in the nucleus at similar density. Fusion peptide bearing a single copy of the motifs was expressed mainly in the cytoplasm [27]. In plant PIPKs, most of MORN motifs repeated for 7-9 times and link sequentially, however, two groups are identified by the structural analysis, which is similar to that of JP1. In addition, when sequentially deleted the MORN motifs from the N-terminus, we observed a shift of the subcellular localization of fusion proteins: all MORN motifs on the membrane and then on the membrane and in the nucleus at similar density and, after deleted all of the MORN motifs, predominantly in the nucleus. These results indicate that the MORN motifs may contribute to the membrane-binding capacity of plant PIPKs and the proposed interaction of plant PIPKs with the target component(s) on the plasma membrane depends on the number and/or sequence of MORN motifs, which is similar to that in JP1.

PIPKs may exit in nucleus and some mammalian PIPK isoforms (including type I and II subfamilies) are concentrated in nucleus, spatially organized in the "nuclear speckles" that are separated from known membrane structure [34]. However, the regulatory mechanism and the function of the PIPKs at speckles is still unknown. Our studies may provide clues for the regulation of spatial organization of the PIPKs. Further study is necessary to elucidate whether plant PIPKs can also localize in some 
specific nuclear fraction and the role of MORN motifs in this possible nuclear locations.

SMET analysis showed that the fusion protein containing the whole MORN region has strong membrane-binding ability, and after sequentially deleting the MORN motifs from the N-terminus, the membrane binding ability of fusion protein decreased; however, even two copies of MORN motif (with the linker) still has membrane-binding ability, while a single copy of MORN motif lacks the membrane-binding ability, suggesting that the interaction of N-terminal MORN motifs with the linker sequence (or even the last one of the MORN motif) is necessary for the that MORN motifs may target membrane through peptide-lipid binding interactions [27]. Fat Western blotting studies showed that the entire MORN motifs region of OsPIPK1 can bind to PA, PI4P, and $\mathrm{PI}(4,5) \mathrm{P}_{2}$ in vitro. Since PI4P and PI $(4,5) \mathrm{P}_{2}$ are corresponding substrate and product of PIPKs, binding of PI4P and PI $(4,5) \mathrm{P}_{2}$ by MORN region indicates that MORN region may be involved in the regulation of OsPIPK1 activity or the determination of substrate specificities.

Similar to PI4P and PI $(4,5) \mathrm{P}_{2}$, PA is not only just structural components of membranes but also chemical signal molecule. PA is a minor lipid representing $1-2 \%$ of the total phospholipid in every eukaryotic cell, and recently evidence is accumulating that PA serves as a second messenger molecule. The level of PA increases within minutes of a wide variety of stress treatments including ethylene, wounding, pathogen elicitors, osmotic and oxidative stress, and abscisic acid (for a review, see [35]). More importantly, in mammalian cells, PA can potently stimulate a type I PIPK activity. Quenching PA function with primary alcohols prevents the synthesis of $\mathrm{PI}(4,5) \mathrm{P}_{2}$ and blocks $\mathrm{PI}(4,5) \mathrm{P}_{2}$ mediated clathrin-coat assembly [36]. This provides a direct evidence for a positive feedback loop between PIPKs and phospholipase D (PLD, the main enzyme to synthesize PA) in mammalian cells. We propose that in plant, there is also a feedback loop between plant PIPKs and phospholipase D because MORN region in OsPIPK1 binds strongly to PA in vitro. Further in vivo study is needed to test this hypothesis.

The possibility that the MORN region could be self-organized and thus form some sort of structure and bind the specific phospholipids can not be excluded. The MORN region is full of negative charge (Figure 3B), which is similar to the N-terminus of human PIPK II $\alpha$ proteins. Future studies on the resolving of the tertiary structure of MORN region would be helpful to solve this problem.

\section{Acknowledgements}

This study was supported by Chinese Academy of Sci- ences and grants from National Natural Sciences Foundation of China (No. 30425029).

\section{References}

1 Martin TF. Phosphoinositide lipids as signaling molecules: common themes for signal transduction, cytoskeletal regulation, and membrane trafficking. Annu Rev Cell Dev Biol 1998; 14:231264.

2 D'Santos CS, Clarke JH, Divecha N. Phospholipid signalling in the nucleus. Een DAG uit het leven van de inositide signalering in de nucleus. Biochim Biophys Acta 1998; 1436:201-232.

3 Toker A. The synthesis and cellular roles of phosphatidylinositide synthesis. Chem Phys Lipids 1998; 98:69-77.

4 Martin TF. Phosphoinositides as spatial regulators of membrane traffic. Curr Opin Neurobiol 1997; 7:331-338.

5 Czech MP. PIP2 and PIP3: complex roles at the cell surface. Cell 2000; 100:603-606.

6 Hurley JH, Meyer T. Subcellular targeting by membrane lipids. Curr Opin Cell Biol 2001; 13:146-152.

7 Toker A, Cantley LC. Signalling through the lipid products of phosphoinositide-3-OH kinase. Nature 1997; 387:673-676.

8 Anderson RA, Boronenkov IV, Doughman SD, Kunz J, Loijens JC. Phosphatidylinositol phosphate kinases, a multifaceted family of signaling enzymes. J Biol Chem 1999; 274:9907-9910.

9 Hinchliffe KA, Ciruela A, Irvine RF. PIPkins1, their substrates and their products: new functions for old enzymes. Biochim Biophys Acta 1998; 1436:87-104.

10 Gary JD, Wurmser AE, Bonangelino CJ, Weisman LS, Emr SD. Fab1p is essential for PtdIns(3)P 5-kinase activity and the maintenance of vacuolar size and membrane homeostasis. J Cell Biol 1998; 143:65-79.

11 McEwen RK, Dove SK, Cooke FT, et al. Complementation analysis in PtdInsP kinase-deficient yeast mutants demonstrates that Schizosaccharomyces pombe and murine Fab1p homologues are phosphatidylinositol 3-phosphate 5-kinases. J Biol Chem 1999; 274:33905-33912.

12 Sbrissa D, Ikonomov OC, Shisheva A. PIKfyve, a mammalian ortholog of yeast Fab1p lipid kinase, synthesizes 5-phosphoinositides. Effect of insulin. J Biol Chem 1999; 274:21589-21597.

13 Fruman DA, Meyers RE, Cantley LC. Phosphoinositide kinases. Annu Rev Biochem 1998; 67:481-507.

14 Tolias KF, Cantley LC. Pathways for phosphoinositide synthesis. Chem Phys Lipids 1999; 98:69-77.

15 Rao VD, Misra S, Boronenkov IV, Anderson RA, Hurley JH. Structure of type IIbeta phosphatidylinositol phosphate kinase: a protein kinase fold flattened for interfacial phosphorylation. Cell 1998; 94:829-839.

16 Xue HW, Pical C, Brearley C, Elge S, Mueller-Roeber B. A plant 126-kDa phosphatidylinositol 4-kinase with a novel repeat structure. Cloning and functional expression in baculovirus-infected insect cells. J Biol Chem 1999; 274:5738-5745.

17 Hong Z, Verma DP. A phosphatidylinositol 3-kinase is induced during soybean nodule organogenesis and is associated with membrane proliferation. Proc Natl Acad Sci USA 1994; 91:96179621.

18 Welters P, Takegawa K, Emr SD, Chrispeels MJ. AtVPS34, a phosphatidylinositol 3-kinase of Arabidopsis thaliana, is an essential protein with homology to a calcium-dependent lipid bind- 
ing domain. Proc Natl Acad Sci USA 1994; 91:11398-11402.

19 Xue HW, Hosaka K, Plesch G, Mueller-Roeber B. Cloning of Arabidopsis thaliana phosphatidylinositol synthase and functional expression in the yeast pis mutant. Plant Mol Biol 2000; 42:757-764.

20 Mikami K, Katagiri T, Iuchi S, Yamaguchi-Shinozaki K, Shinozaki K. A gene encoding phosphatidylinositol-4-phosphate 5-kinase is induced by water stress and abscisic acid in Arabidopsis thaliana. Plant J 1998; 15:563-568.

21 Shi J, Gonzales RA, Bhattacharyya MK. Characterization of a plasma membrane-associated phosphoinositide-specific phospholipase C from soybean. Plant J 1995; 8:381-390.

22 Hirayama T, Ohto C, Mizoguchi T, Shinozaki K. A gene encoding a phosphatidylinositol-specific phospholipase $\mathrm{C}$ is induced by dehydration and salt stress in Arabidopsis thaliana. Proc Natl Acad Sci USA 1995; 92:3903-3907.

23 Berdy SE, Kudla J, Gruissem W, Gillaspy GE. Molecular characterization of At5PTase1, an inositol phosphatase capable of terminating inositol trisphosphate signaling. Plant Physiol 2001; 126: $801-810$.

24 Lin WH, Ye R, Ma H, Xu ZH, Xue HW. DNA chip-based expression profile analysis indicates involvement of the phosphatidylinositol signaling pathway in multiple plant responses to hormone and abiotic treatments. Cell Res 2004; 14:34-45.

25 Stevenson-Paulik J, Odom AR, York JD. Molecular and biochemical characterization of two plant inositol polyphosphate 6-/3-/5-kinase. J Biol Chem 2002; 277:42711-42718.

$26 \mathrm{Xu}$ J, Brearley CA, Lin WH, Wang Y, Ye R, Mueller-Roeber B, $\mathrm{Xu} \mathrm{ZH}$, Xue HW. A role of Arabidopsis inositol polyphosphate kinase, AtIPK2alpha, in pollen germination and root growth. Plant Physiol 2005; 137:94-103.

27 Takeshima H, Komazaki S, Nishi M, Ino M , Kangawa K. Junctophilins: a novel family of junctional membrane complex proteins. Mol Cell 2000; 6:11-22.
$28 \mathrm{Ma} \mathrm{H}, \mathrm{Xu}$ SP, Luo D, Xu ZH, Xue HW. OsPIPK 1, a rice phosphatidylinositol monophosphate kinase, regulates rice heading by modifying the expression of floral induction genes. Plant Mol Biol 2004; 54:295-310.

29 Lou Y, Ma H, Lin WH, et al. The highly charged region of plant $\beta$-type phosphatidylinositol 4-kinase is involved in membrane targeting and phospholipid binding. Plant Molecular Biology 2006; in press.

30 Sambrook J, Russellm RW. Molecular cloning 2001: a laboratory manual, 3rd ed. Cold Spring, NY: Cold Spring Harbor Laboratory Press.

31 Sohlenkamp C, Wood CC, Roeb GW , Udvardi MK. Characterization of Arabidopsis AtAMT2, a high-affinity ammonium transporter of the plasma membrane. Plant Physiol 2002; 130:1788-1796.

32 Stevenson JM, Perera IY, Boss WF. A phosphatidylinositol 4-kinase pleckstrin homology domain that binds phosphatidylinositol 4-monophosphate. J Biol Chem 1998; 273:22761-22767.

33 Mueller-Roeber B, Pical C. Inositol phospholipid metabolism in Arabidopsis. Characterized and putative isoforms of inositol phospholipid kinase and phosphoinositide-specific phospholipase C. Plant Physiol 2002; 130:22-46.

34 Boronenkov IV, Loijens JC, Umeda M, Anderson RA. Phosphoinositide signaling pathways in nuclei are associated with nuclear speckles containing pre-mRNA processing factors. Mol Biol Cell 1998; 9:3547-3560.

35 Munnik, T. Phosphatidic acid: an emerging plant lipid second messenger. Trends Plant Sci 2001; 6:227-233.

36 Arneson LS, Kunz J, Anderson RA, Traub LM. Coupled inositide phosphorylation and phospholipase D activation initiates clathrin-coat assembly on lysosomes. J Biol Chem 1999; 274:1779417805.

37 Kyte J, Doolittle RF. A simple method for displaying the hydropathic character of a protein. J Mol Biol 1982; 157:105-132.

Edited by Sheng Luan 